\title{
TXNDC17 Protein Overexpression in Uterine Papillary Serous Carcinoma is Associated with Chemoresistance
}

\section{Qiaohua Peng}

Women's Hospital School Of Medicine Zhejiang University

Yan Yu

Women's Hospital School Of Medicine Zhejiang University

\section{Xiaodong Cheng}

Women's Hospital School Of Medicine Zhejiang University

\section{Xinyu Wang}

Women's Hospital School Of Medicine Zhejiang University

Weiguo Lu

Women's Hospital School Of Medicine Zhejiang University

\section{Xing Xie}

Women's Hospital School Of Medicine Zhejiang University

Songfa Zhang ( $\nabla$ zhangsongfa@zju.edu.cn )

Women's Hospital School Of Medicine Zhejiang University

\section{Research Article}

Keywords: TXNDC17, Autophagy, Uterine Papillary Serous Carcinoma, Drug Resistance

Posted Date: March 9th, 2021

DOl: https://doi.org/10.21203/rs.3.rs-215151/v1

License: (c) (1) This work is licensed under a Creative Commons Attribution 4.0 International License. Read Full License 


\section{Abstract}

Background: TXNDC17(thioredoxin domain containing 17 protein) has primarily been associated with ovarian cancer. It concurs resistance to paclitaxel by inducing autophagy. However, limited information exists on its role in uterine papillary serous carcinoma (UPSC). This study aimed to analyze clinicopathological characteristics of patients with UPSC in China, and investigate roles of TXNDC17 protein in UPSC chemoresistance and prognosis.

Methods: Fifty-five patients with UPSC treated in women's hospital school of medicine Zhejiang university from 2003 to 2016 were analysed retrospectively. Immunohistochemistry (IHC) were performed to TXNDC17 and BECN1 (Beclin 1 protein, a key regulator of autophagy) protein expression respectively.

Results: A total of 55 UPSC patients of median age ( 63.3 years), $43.7 \%$ with late stages (stage III/IV), and $48.6 \%$ exhibiting more than half of myometrium invasion were enrolled. Notably, 28 (50.9\%) patients had TXNDC17 protein overexpression associated with chemoresistance and high BECN1 expression. BECN1 overexpression occurred at an advanced stage and was characterized by deep myometrial invasion. Of note, advanced stage, deep myometrial invasion, BECN1, and TXNDC17 overexpression were significantly associated with a poor outcome. Besides, co-expression of TXNDC17 and BECN1 occurred at an advanced stage causing deep myometrial invasion and chemoresistance thereby resulted in the shortest survival in UPSC. The multivariate proportional hazards model revealed that myometrial invasion and TXNDC17 overexpression were independent prognostic factors.

Conclusion: This study showed that TXNDC17 overexpression, through induces autophagy, and consequently results in chemoresistance and poor survival in UPSC patients. TXDNC17 may be a potential predictor or target in UPSC therapeutics.

\section{Background}

Endometrial carcinoma is the sixth most common cancer and the 14th leading cause of cancer death in women globally $₫ 1 \rrbracket$. Endometrial carcinoma is classified into two subtypes based on different clinicopathological characteristics and morphological appearance. Type I is estrogen-related and are mostly endometrioid adenocarcinomas comprising of several endometrial cancers derived from endometrial hyperplasia. It is associated with unopposed estrogen stimulation. Type II is estrogenindependent and are predominant serous carcinomas associated with atrophic endometrium. It arises from intraepithelial carcinoma, a precancerous lesion $₫ 2 \rrbracket$.

Uterine papillary serous carcinoma (UPSC) is a typical type II endometrial carcinoma mostly associated with an aggressive clinical course and poor prognoses. It is histologically and clinically more similar to ovarian serous carcinoma $₫ 3 \otimes$. UPSC represents about $10 \%$ of endometrial carcinomas, however, it has caused over $50 \%$ of all deaths and recurrences caused by endometrial carcinomas $₫ 4$. Comprehensive surgical staging and platinum/taxane-based adjuvant chemotherapy are recommended for UPSC patients following guidelines from the Society of Gynecologic Oncology (SGO). This is because UPSC 
exhibits similar characteristics as serous epithelial ovarian carcinoma冈3冈. Poor outcomes of UPSC patients have urgently inspired investigations of new and effective UPSC treatment modalities. However, the response rates to chemotherapy and disease-specific survival rate are lower for UPSC than for ovarian carcinoma patients. UPSC is thus considered chemoresistant and mainly causes low survival rates $₫ 5 \rrbracket$. Besides, studies indicate that UPSC patients treated with paclitaxel with or without platinum show between $60-80 \%$ response rates $₫ 6 \rrbracket$. Of note, most of these responses are not durable and have not translated into improved survival rates. The poor outcomes of the patients require in-depth studies to develop a novel UPSC treatment. Drug resistance is considered the main cause of UPSC therapy failure. The underlying mechanism for UPSC chemoresistance has not been established. Also, there are no definite biomarkers for UPSC chemoresistance. In our previous studies, we reported that TXNDC17 is associated with paclitaxel resistance in ovarian cancer》7囚.

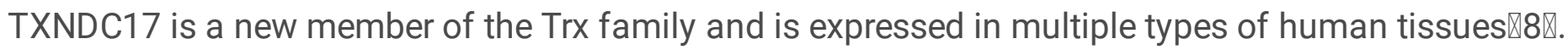
Limited reports exist on the function of TXNDC17. Recent studies have reported that TXNDC17 is involved in the TNF signaling pathway. The pathway is associated with autophagy by interacting with the dynein light chain LC8 protein $₫ 9$. Our previous studies reported that TXNDC17 induce autophagy and consequently promotes resistance of ovarian cancer cells to paclitaxel by regulating BECN1 expression Q7D. In addition, STAT3-dependent TXNDC17 expression mediates paclitaxel resistance by inducing autophagy in human colorectal cancer cells $\otimes 10 \bigotimes$. Autophagy can confer cells with the ability to adapt and thrive in new hostile environments by protecting cancer cells from undesired threats in the late tumor stages. Notably, when autophagy is regulated, some cancer cells acquire the ability to subsist stress induced by anticancer treatment $₫ 11$. However, the biological function of TXNDC17 in UPSC has not been described. Here, we hypothesize that TXNDC17 plays a vital role in disease aggression and UPSC chemoresistance by regulating autophagy.

First, we investigated TXNDC17 expression in 55 UPSC patients through immunohistochemistry analysis. The co-expression of TXNDC17 and BECN1 in the patients was determined. Moreover, we analyzed the relationship between TXNDC17 expression and clinicopathological features including the chemoresistance of UPSC. Therefore, this study purposed to investigate the prognostic value of TXNDC17 and highlight a new strategy for therapeutic intervention in UPSC patients.

\section{Methods}

1,007 patients diagnosed with endometrial carcinoma who were operated at Women's Hospital, Zhejiang University from January 2003 to January 2016 were enrolled. Notably, 86 patients were diagnosed with UPSC which accounted for about $8.5 \%$. The uterine specimens after surgery were diagnosed by two pathologists separately and any dispute in diagnosis was verified by a different senior pathologist through a final diagnosis. From the 86 patients, some uterine tissues were not included for this study because satisfactory specimens could not be obtained or they lacked useful information. A total of 55 cases of UPSC were collected. Patients were staged following the FIGO surgical criteria. Progression-free survival (PFS) and overall survival (OS) were calculated from the date of the surgical diagnosis as the 
primary clinical endpoint evaluation. Death certificate information was obtained when there was no information about survival in the medical record. A patient was censored after her last contact if the information was not available.

Clinicopathological information including age at the time of diagnosis, weight, major symptoms, myometrial invasion, a histologic subtype of UPSC (pure or mixed), lymph node status, FIGO (International Federation of Gynecology and Obstetrics) stage, histologic grade, ER/PR/P53/Ki-67 level, therapeutic regimen, and history of medicines. The patients were placed into two groups based on their sensitivity to platinum-based combination chemotherapy. Chemoresistant group was defined as a recurrence fewer than 6 months after platinum chemotherapeutic. The chemosensitive group was defined as a recurrence of 6 months or later after platinum therapy.

Formalin fixed and paraffin embedded sections were dewaxed in xylene, rehydrated in graded ethanol and saturated with endogenous peroxides. Paraffin sections were cultured with anti-TXNDC17 (Abcam, ab121725) antibody which dilute to 1:150 or anti-BECN1 (Abcam, ab51031) antibody dilute 1:100. Two serial sections of the same paraffin embedded block and the same patient were stained with antiTXNDC17 and anti-BECN1 antibodies respectively. The staining samples were displayed by Dako real envision detection system (Dako Diagnostica, K5007). Section slides were stained with hematoxylin, dehydrated and fixed. The negative control groups did not contain primary antibody. TXNDC17 and BECN1 staining were semi quantitatively analyzed in 5 microscope fields. The intensity of immunostaining was graded as follows: weak, $1+$; moderate, $2+$; strong, $3+$ or very strong, $4+$. In each microscopic the area of positive were classified as follows: 0 to $25 \%, 1+; 25$ to $50 \%, 2+; 50$ to $75 \%$, $3+$, or 75 to $100 \%, 4+$. Multiply the two fractions to get the sum between 5 and 80 . The "low expression" means the sum from 0 to 42 , and the "high expression" means the sum from 43 to 80 .

We used $\chi 2$ tests to estimate the prognostic factors, and Kaplan-Meier survival curves were performed to evaluate progression-free survival (PFS) and overall survival (OS). The survival curves were compared by log-rank test. We use Cox's proportional hazards regression to model survival with TXNDC17

overexpression and the clinicopathological factors associated with prognosis. SPSS 20.0 software (IBM Corp., Armonk, NY, USA) were used to statistical analyses. P values less than or equal to 0.05 were considered statistically significant.

\section{Results}

The patients were of median age (63.3 years) ranging from 49-79 years. The median BMI (Body Mass Index) was $23.89 \mathrm{~kg} / \mathrm{m}^{2}$ (range 18.73-32.03). The major presenting symptom was abnormal vaginal bleeding $(87.7 \%, 48 / 55)$. Besides, three patients were initially presented with vaginal discharge, four patients exhibited intrauterine mass diagnosis by gynecological ultrasound, and one patient showed bellyache. Notably, 15 patients had hypertension complications, two patients had diabetes, and five patients had both hypertension and diabetes. Moreover, four patients had a history of other cancers including breast cancer (1), kidney cancer (1) and, ovarian cancer (2). 
From the 55 patients, 24 (43.6\%) exhibited stage I (19 IA, 5 IB), 7 (12.7\%) stage II (4 IIA, 3 IIB), 17 (31\%) stage III (4 IIIA, 3IIIB, 10IIIC), and 7 (12.7\%) stage IV (3IVA, 4IVB) of UPSC. Altogether $56.3 \%$ showed earlystage (stage I/II) and $43.7 \%$ showed late-stage (stage III/IV) UPSC infection. Notably, 33 tumors invaded less than half of the myometrium, whereas 18 tumors invaded more than half of, however 4 patients had no myometrial invasion. Exactly 14 tumors (25.4\%) exhibited LVSI (Lymph-vascular space invasion), 33 patients $(60 \%)$ had P53(Tumor Suppressor Protein p53) overexpression, 34 patients $(61.8 \%)$ had Ki-67(Ki67 Antigen) overexpression. Moreover, positive ER(estrogen receptor) expression was detected in 14 cases $(25 \%)$ and $\mathrm{PR}$ (progesterone receptor) expression in 7 cases $(12.7 \%)$. All patients were subjected to underwent exploratory surgery, hysterectomy, bilateral salpingo-oophorectomy, omentectomy, pelvic lymphadenectomy, and/or para-aortic lymphadenectomy, and platinum/taxane-based adjuvant chemotherapy after surgery.

A total of 28 (50.9\%) patients had TXNDC17 protein overexpression through IHC (Figure1). The clinicopathological features due to TXNDC17 overexpression are summarized in Table 1. High TXNDC17 expression was significantly correlated with chemoresistance $(P=0.014)$ and higher $B E C N 1$ levels $(P=0.023)$. Furthermore, we determined BECN1 expression in UPSC through IHC, 29 (52.7\%) patients had BECN1 protein overexpression (Figure1). The clinicopathological features from BECN1 overexpression are summarized in Table 2. Patients showing BECN1 overexpression were more likely to exhibit advanced stage $(P=0.028)$ and deep myometrial invasion $(P=0.029)$ compared with BECN1 negative patients. BECN1 overexpression was reported in $71.4 \%$ of UPSC patients showing advanced disease stage compared to $41 \%$ of BECN1 negative patients. Besides, patients with BECN1 overexpression (72.2\%) were more likely to show deep myometrial invasion than BECN1 negative patients (43\%).

In order to explore the role of TXNDC17 in autophagy, we divided the patients into 4 groups including TXNDC17high/BECN1high, TXNDC17high/BECN1low, TXNDC17low/BECN1high, and TXNDC17low/BECN1low. Compared with other groups, TXNDC17high /BECN1 high groups had an advanced stage $(P=0.013)$, depth myometrial invasion $(P=0.009)$, and chemotherapy resistance $(\mathrm{P}=0.006)$ (Table 3). The results indicated that TXNDC17 and BECN1 proteins higher expression are closely linked with malignant features in UPSC..

Results from univariate survival analysis of TXNDC17 protein expression and other clinicopathological factors are shown in Table 4. The median follow-up time for all the patients was 45.7 months (range 7120 months). Lower outcome (Kaplan-Meier) was observed in TXNDC17 positive patients than negative patients (Figure 2). Kaplan-Meier survival curves showed that UPSC with high TXNDC17 expression exhibited significantly lower progression-free survival ( $P F S, P=0.028$ ) and overall survival ( $O S, P=0.008)$. The 5-year overall survival rate for early and advanced stage patients was $88.2 \%$ and $61.9 \%$. Besides, the survival rate for the superficial and deep myometrial invasion was $86.5 \%$ and $50.0 \%$ respectively. Deep myometrial invasion and advanced stage were significantly associated with a shorter OS (Figure 2a) and PFS (Figure 2b). Moreover, high BECN1 expression was associated with a lower outcome (Figure 2). When patients were divided into four groups (TXDNC17 $7^{\text {high }} / \mathrm{BECN} 1^{\text {high }}$, TXDNC17 $^{\text {high }} / \mathrm{BECN} 1^{\text {low }}$, $\mathrm{TXDNC} 7^{\text {low }} / \mathrm{BECN} 1^{\text {high }}$, TXDNC17 $^{\text {low }} / \mathrm{BECN} 1^{\text {low }}$ ) patients with $\mathrm{TXDNC}^{\text {high }} / \mathrm{BECN} 1^{\text {high }}$ displayed the 
shortest survival in UPSC (Figure 3). Multivariate analysis showed that myometrial invasion ( $P=0.011$ ) and TXNDC17 expression ( $P=0.012)$ were independent prognostic factors for overall survival (Table 5$)$. These findings suggested that a potential linkage exists among TXNDC17, autophagy, and malignant features of UPSC.

\section{Discussion}

Uterine papillary serous carcinoma (UPSC) is a rare type of endometrial cancer. Besides, highly aggressive endometrial carcinoma is characterized by early metastatic spread, high recurrence rate, and poor prognosis compared with type I endometrial carcinoma. Previous studies have evaluated several prognostic factors of UPSC such as stage, age, depth of myometrial, tumor size, lymphatic vascular space invasion (LVSI), grade, tumor markers, and cytoreductive surgery, however, their findings are inconsistent $₫ 12 \bigotimes$. Of note, most findings indicate that myometrial invasion and tumor stage highly influence the prognosis of UPSC patients. Reports by Black and his colleagues indicated that myometrial invasion and tumor stage were the main factors for UPSC prognosis $\triangle 13 \otimes$. Elsewhere, a study showed that

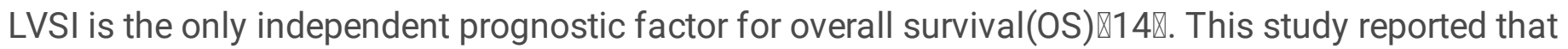
myometrial invasion and FIGO stages were risk factors for a worse UPSC prognosis, however, there was no significant difference in LVSI, the tumor grade, P53 and Ki-67 expression. Additionally, the multivariate analysis reported myometrial invasion as an independent prognostic for poor survival of UPSC.

Considering the contradicting results from previous studies, we supposed that a lack of patient numbers and limited follow-up time may affect the accuracy of statistical results. Therefore, larger patient samples are needed for a similar study to clarify the UPSC prognosis.

Notably, UPSC has similar characteristics with the ovarian serous carcinoma compared to type I endometrial carcinoma. They include the tendency to metastasize on the peritoneal surfaces and lymph node, occurrence of ascites, and upper abdominal invasionヌ3区. However, UPSC is potentially chemoresistant. In our previous research, we reported that high TXNDC17 expression is associated with poorer prognosis of ovarian cancer patients. Moreover, TXNDC17 promotes paclitaxel resistance by inducing autophagy in ovarian cancer囚7区. This study reports that TXDNC17 is overexpressed in UPSC thereby is associated with chemoresistance and is a risk factor for a worse UPSC prognosis.

Besides, TXNDC17 is a ubiquitous cytosolic protein expressed in mammalian tissues. It contains Trx-like active center proteins, functions as a disulfide reductase and peroxidase, and maintains redox homeostasis in cells $\mathbb{8} 8$. Limited information on the function of TXNDC17 exists, however, recent studies have reported that TXNDC17 is involved in the TNF-asignal transduction pathway (a stimulating pathway for cellular autophagy) regulation $\otimes 15 \Downarrow$. Moreover, autophagy has been reported as one of the modalities of cell death occurring after chemotherapy and is an underlying mechanism for chemoresistance in tumor cells $\triangle 16 \bigotimes$. We examined the association of the expression between TXDNC17 and BECN1, a key regulator of autophagy, to determine whether autophagy is associated with TXNDC17-related poor prognosis and chemoresistance. Of note, a significant positive correlation between TXDNC17 and BECN1 expression in UPSC was observed. 
Furthermore, TXNDC17 was reported as a risk factor for poor UPSC prognosis and is remarkably correlated to chemoresistance and higher BECN1 expression. BECN1 expression in UPSC was determined and patients showing BECN1 overexpression were more likely to develop advanced stage and depth myometrial invasion compared to BECN1 negative patients. Also, patients with TXDNC1 $7^{\text {high }} / \mathrm{BECN} 1$ high exhibited the shortest survival in UPSC, and patients with TXDNC17 $7^{\text {high }} / \mathrm{BECN} 11^{\text {high }}$ were more likely to have an advanced stage, depth myometrial invasion, and chemoresistance. Moreover, multivariate analysis showed that TXNDC17 expression is an independent prognostic factor for overall survival. This confirmed that TXNDC17 is associated with poor prognosis and chemoresistance by inducing autophagy in UPSC.

Autophagy can cause type II programmed cell death. Effective activation of autophagy confers stress tolerance and maintains cell survival, however excessive or sustained autophagy potentially induce cell death $\triangle 17$. Besides, autophagy can provide cells with the ability to adapt and thrive in new hostile adverse environments by protecting cancer cells from undesired threats in late tumor stages $₫ 18 \bigotimes$. Also, autophagy is associated with recurrence, metastasis, and chemotherapy resistance in many cancer types \19-21》. Previously, we reported that autophagy maintains the stemness of ovarian cancer stem cells $₫ 22 \rrbracket$ and TXNDC17 promotes paclitaxel resistance by inducing autophagy in ovarian cancer囚7囚. Other studies also have reported that STAT3-dependent TXNDC17 expression mediates paclitaxel resistance by inducing autophagy in human colorectal cancer cells $₫ 10 \bigotimes$. Additionally, suberoylanilide hydroxamic acid has been reported to sensitize neuroblastoma to paclitaxel by inhibiting TXNDC17-mediated autophagy Q23区. In this study, we observed TXNDC17 and BECN1 co-expression in some UPSC and TXNDC17 overexpression caused higher BECN1 expression, therefore, it is associated with poor patient prognosis. Moreover, TXNDC17 expression was an independent prognostic factor for overall survival and associated with chemoresistance and is thus a potential target in UPSC therapeutics. Combining the novel targeted therapies that stimulate autophagy with conventional anticancer drugs may provide a new treatment for UPSC, therefore should further be investigated.

\section{Conclusions}

Our study demonstrate for the first time that high TXNDC17 expression is associated with poor prognosis of UPSC. TXNDC17, through autophagy participation, promotes an aggressive clinical course causing chemoresistance and poor UPSC outcome. Findings from this study suggest that TXDNC17 may be a potential therapeutic target in refractory UPSC patients. However, the underlying mechanisms should be further evaluated by randomized clinical trials.

\section{Abbreviations}

TXNDC17: thioredoxin domain containing 17 protein; UPSC: uterine papillary serous carcinoma; PFS: Progression-free survival; OS: overall survival; FIGO: International Federation of Gynecology and Obstetrics; LVSI: Lymph-vascular space invasion; 


\section{Declarations}

1. Lortet-Tieulent J, Ferlay J, Bray F, Jemal A: International Patterns and Trends in Endometrial Cancer Incidence, 1978-2013. J Natl Cancer Inst 2018, 110(4):354-361.

2. Setiawan VW, Yang HP, Pike MC, McCann SE, Yu H, Xiang YB, Wolk A, Wentzensen N, Weiss NS, Webb PM et al: Type I and II endometrial cancers: have they different risk factors? J CLIN ONCOL 2013, 31(20):2607-2618.

3. Lin JF, Muniz K, Sukumvanich P, Gehrig P, Beriwal S, Kelley JL, Edwards RP, Olawaiye AB: Survival advantage associated with multimodal therapy in women with node-positive (stage-IIIC) uterine papillary serous carcinoma: a National Cancer Database study. BJOG 2016, 123(11):1846-1852.

4. Fader AN, Boruta D, Olawaiye AB, Gehrig PA: Uterine papillary serous carcinoma: epidemiology, pathogenesis and management. Curr Opin Obstet Gynecol 2010, 22(1):21-29.

5. Ren Y, Wang H, Zhou X, Yang W, Huang X, Lu Y, Shi D: Clinicopathological Characteristics and Her2/neu Status in Chinese Patients with Uterine Papillary Serous Carcinoma. ISRN Obstetrics and Gynecology 2011, 2011:1-8.

6. Tortorella L, Langstraat CL, Weaver AL, McGree ME, Bakkum-Gamez JN, Dowdy SC, Cliby WA, Keeney GL, Sherman ME, Weroha SJ et al: Uterine serous carcinoma: Reassessing effectiveness of platinumbased adjuvant therapy. GYNECOL ONCOL 2018, 149(2):291-296.

7. Zhang SF, Wang XY, Fu ZQ, Peng QH, Zhang JY, Ye F, Fu YF, Zhou CY, Lu WG, Cheng XD et al: TXNDC17 promotes paclitaxel resistance via inducing autophagy in ovarian cancer. AUTOPHAGY 2015, 11(2):225-238.

8. Zhen Z, Yang K, Ye L, You Z, Chen R, Liu Y, He Y: Suberoylanilide hydroxamic acid sensitizes neuroblastoma to paclitaxel by inhibiting thioredoxin-related protein 14-mediated autophagy. CANCER SCI 2017, 108(7):1485-1492.

9. Pader I, Sengupta R, Cebula M, Xu J, Lundberg JO, Holmgren A, Johansson K, Elias SJA: Thioredoxinrelated protein of $14 \mathrm{kDa}$ is an efficient L-cystine reductase and S-denitrosylase. Proceedings of the National Academy of Sciences 2014, 111(19):6964-6969.

10. Zhang Z, Wang A, Li H, Zhi H, Lu F: RETRACTED: STAT3-dependent TXNDC17 expression mediates Taxol resistance through inducing autophagy in human colorectal cancer cells. GENE 2016, 584(1):75-82.

11. Galan-Acosta L, Xia H, Yuan J, Vakifahmetoglu-Norberg H: Activation of chaperone-mediated autophagy as a potential anticancer therapy. AUTOPHAGY 2015, 11(12):2370-2371.

12. Wang Y, Yu M, Yang J, Cao D, Shen K, Lang J: Clinicopathological and survival analysis of uterine papillary serous carcinoma: a single institutional review of 106 cases. 2018, Volume 10:4915-4928.

13. Black C, Feng A, Bittinger S, Quinn M, Neesham D, McNally O: Uterine Papillary Serous Carcinoma A Single-Institution Review of 62 Cases. INT J GYNECOL CANCER 2016, 26(1):133-140.

14. Winer I, Ahmed QF, Mert I, Bandyopadhyay S, Cote M, Munkarah AR, Hussein Y, Al-Wahab Z, Elshaikh MA, Alosh B et al: Significance of Lymphovascular Space Invasion in Uterine Serous Carcinoma: 
What Matters More; Extent or Presence? INT J GYNECOL PATHOL 2015, 34(1):47-56.

15. Jeong W, Jung Y, Kim H, Park SJ, Rhee SG: Thioredoxin-related protein 14, a new member of the thioredoxin family with disulfide reductase activity: Implication in the redox regulation of TNF-alpha signaling. FREE RADICAL BIO MED 2009, 47(9SI):1294-1303.

16. Li Y, Lei Y, Yao N, Wang C, Hu N, Ye W, Zhang D, Chen Z: Autophagy and multidrug resistance in cancer. CHIN J CANCER 2017, 52(52).

17. Wang K: Autophagy and apoptosis in liver injury. CELL CYCLE2015, 14(11):1631-1642.

18. Amaravadi R, Kimmelman AC, White $E$ : Recent insights into the function of autophagy in cancer. GENE DEV 2016, 30(17):1913-1930.

19. White $\mathrm{E}:$ The role for autophagy in cancer. J CLIN INVEST 2015, 125(1):42-46.

20. Onorati AV, Dyczynski M, Ojha R, Amaravadi RK: Targeting autophagy in cancer. CANCER-AM CANCER SOC 2018, 124(16):3307-3318.

21. Lei Y, Zhang D, Yu J, Dong H, Zhang J, Yang S: Targeting autophagy in cancer stem cells as an anticancer therapy. CANCER LETT 2017, 393:33-39.

22. Peng Q, Qin J, Zhang $Y$, Cheng $X$, Wang $X$, Lu W, Xie X, Zhang S: Autophagy maintains the stemness of ovarian cancer stem cells by FOXA2. J Exp Clin Cancer Res 2017, 36(1):171.

23. Zhen Z, Yang K, Ye L, You Z, Chen R, Liu Y, He Y: Suberoylanilide hydroxamic acid sensitizes neuroblastoma to paclitaxel by inhibiting thioredoxin-related protein 14-mediated autophagy. CANCER SCI 2017, 108(7):1485-1492.

\section{References}

1. Lortet-Tieulent J, Ferlay J, Bray F, Jemal A: International Patterns and Trends in Endometrial Cancer Incidence, 1978-2013. J Natl Cancer Inst 2018, 110(4):354-361.

2. Setiawan VW, Yang HP, Pike MC, McCann SE, Yu H, Xiang YB, Wolk A, Wentzensen N, Weiss NS, Webb PM et al: Type I and II endometrial cancers: have they different risk factors? J CLIN ONCOL 2013, 31(20):2607-2618.

3. Lin JF, Muniz K, Sukumvanich P, Gehrig P, Beriwal S, Kelley JL, Edwards RP, Olawaiye AB: Survival advantage associated with multimodal therapy in women with node-positive (stage-IIIC) uterine papillary serous carcinoma: a National Cancer Database study. BJOG 2016, 123(11):1846-1852.

4. Fader AN, Boruta D, Olawaiye AB, Gehrig PA: Uterine papillary serous carcinoma: epidemiology, pathogenesis and management. Curr Opin Obstet Gynecol 2010, 22(1):21-29.

5. Ren Y, Wang H, Zhou X, Yang W, Huang X, Lu Y, Shi D: Clinicopathological Characteristics and Her2/neu Status in Chinese Patients with Uterine Papillary Serous Carcinoma. ISRN Obstetrics and Gynecology 2011, 2011:1-8.

6. Tortorella L, Langstraat CL, Weaver AL, McGree ME, Bakkum-Gamez JN, Dowdy SC, Cliby WA, Keeney GL, Sherman ME, Weroha SJ et al: Uterine serous carcinoma: Reassessing effectiveness of platinumbased adjuvant therapy. GYNECOL ONCOL 2018, 149(2):291-296. 
7. Zhang SF, Wang XY, Fu ZQ, Peng QH, Zhang JY, Ye F, Fu YF, Zhou CY, Lu WG, Cheng XD et al: TXNDC17 promotes paclitaxel resistance via inducing autophagy in ovarian cancer. AUTOPHAGY 2015, 11(2):225-238.

8. Zhen Z, Yang K, Ye L, You Z, Chen R, Liu Y, He Y: Suberoylanilide hydroxamic acid sensitizes neuroblastoma to paclitaxel by inhibiting thioredoxin-related protein 14-mediated autophagy. CANCER SCI 2017, 108(7):1485-1492.

9. Pader I, Sengupta R, Cebula M, Xu J, Lundberg JO, Holmgren A, Johansson K, Elias SJA: Thioredoxinrelated protein of $14 \mathrm{kDa}$ is an efficient L-cystine reductase and S-denitrosylase. Proceedings of the National Academy of Sciences 2014, 111(19):6964-6969.

10. Zhang Z, Wang A, Li H, Zhi H, Lu F: RETRACTED: STAT3-dependent TXNDC17 expression mediates Taxol resistance through inducing autophagy in human colorectal cancer cells. GENE 2016, 584(1):75-82.

11. Galan-Acosta L, Xia H, Yuan J, Vakifahmetoglu-Norberg H: Activation of chaperone-mediated autophagy as a potential anticancer therapy. AUTOPHAGY 2015, 11(12):2370-2371.

12. Wang Y, Yu M, Yang J, Cao D, Shen K, Lang J: Clinicopathological and survival analysis of uterine papillary serous carcinoma: a single institutional review of 106 cases. 2018, Volume 10:4915-4928.

13. Black C, Feng A, Bittinger S, Quinn M, Neesham D, McNally O: Uterine Papillary Serous Carcinoma A Single-Institution Review of 62 Cases. INT J GYNECOL CANCER 2016, 26(1):133-140.

14. Winer I, Ahmed QF, Mert I, Bandyopadhyay S, Cote M, Munkarah AR, Hussein Y, Al-Wahab Z, Elshaikh MA, Alosh B et al: Significance of Lymphovascular Space Invasion in Uterine Serous Carcinoma: What Matters More; Extent or Presence? INT J GYNECOL PATHOL 2015, 34(1):47-56.

15. Jeong W, Jung Y, Kim H, Park SJ, Rhee SG: Thioredoxin-related protein 14 , a new member of the thioredoxin family with disulfide reductase activity: Implication in the redox regulation of TNF-alpha signaling. FREE RADICAL BIO MED 2009, 47(9SI):1294-1303.

16. Li Y, Lei Y, Yao N, Wang C, Hu N, Ye W, Zhang D, Chen Z: Autophagy and multidrug resistance in cancer. CHIN J CANCER 2017, 52(52).

17. Wang K: Autophagy and apoptosis in liver injury. CELL CYCLE2015, 14(11):1631-1642.

18. Amaravadi R, Kimmelman AC, White $E$ : Recent insights into the function of autophagy in cancer. GENE DEV 2016, 30(17):1913-1930.

19. White E: The role for autophagy in cancer. J CLIN INVEST 2015, 125(1):42-46.

20. Onorati AV, Dyczynski M, Ojha R, Amaravadi RK: Targeting autophagy in cancer. CANCER-AM CANCER SOC 2018, 124(16):3307-3318.

21. Lei Y, Zhang D, Yu J, Dong H, Zhang J, Yang S: Targeting autophagy in cancer stem cells as an anticancer therapy. CANCER LETT 2017, 393:33-39.

22. Peng $Q$, Qin J, Zhang $Y$, Cheng $X$, Wang $X$, Lu W, Xie X, Zhang S: Autophagy maintains the stemness of ovarian cancer stem cells by FOXA2. J Exp Clin Cancer Res 2017, 36(1):171. 
23. Zhen Z, Yang K, Ye L, You Z, Chen R, Liu Y, He Y: Suberoylanilide hydroxamic acid sensitizes neuroblastoma to paclitaxel by inhibiting thioredoxin-related protein 14-mediated autophagy. CANCER SCI 2017, 108(7):1485-1492.

\section{Tables}

Due to technical limitations, tables are only available as a download in the Supplemental Files section.

\section{Figures}
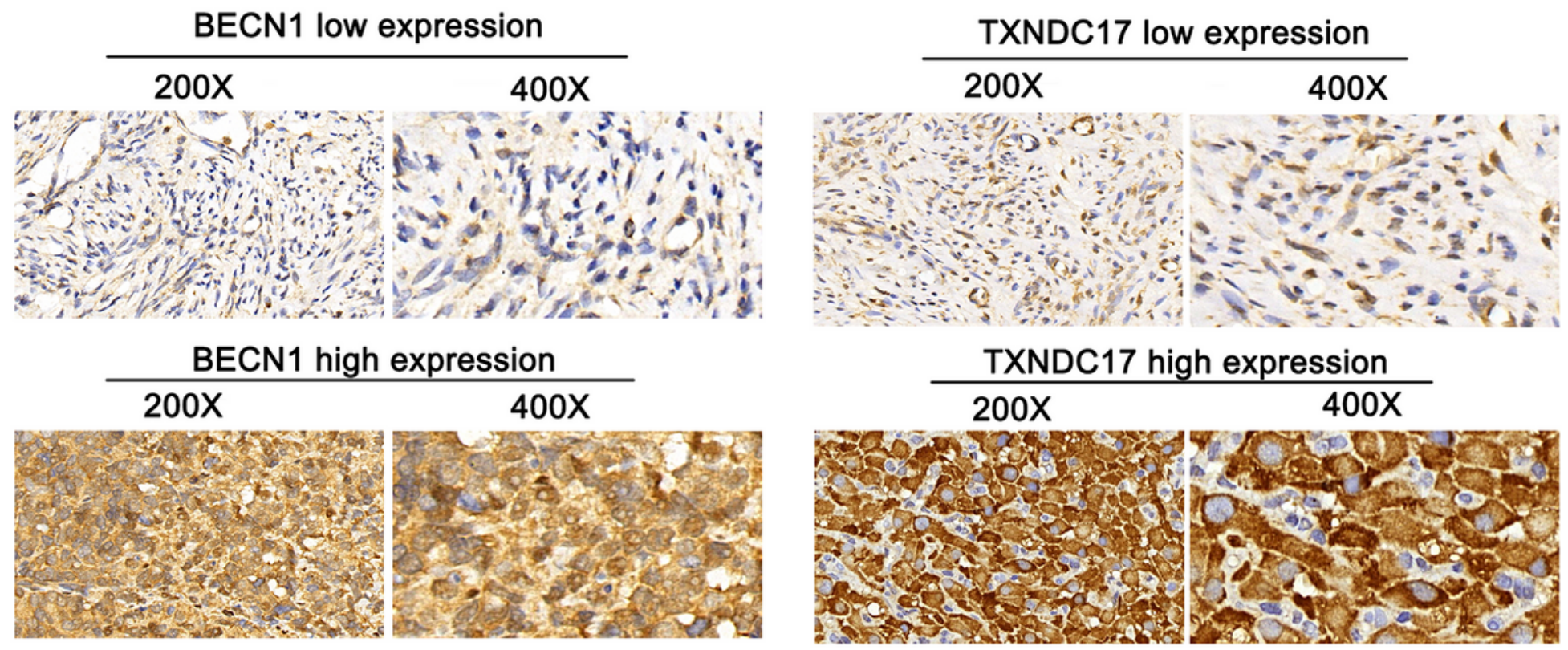

\section{Figure 1}

Immunohistochemistry of BECN1 and TXNDC17 expression in UPSC patients:Two serial sections from the same paraffin-embedded block from 55 UPSC patients were used for detection using anti-TXNDC17 and anti-BECN1 antibodies, respectively. Representative TXNDC17 and BECN1 staining from low expression and high expression tissue are shown at 200X and 400X magnifications. TXNDC17, thioredoxin domain containing 17 protein; BECN1, Beclin 1 protein; UPSC, uterine papillary serous carcinoma. 


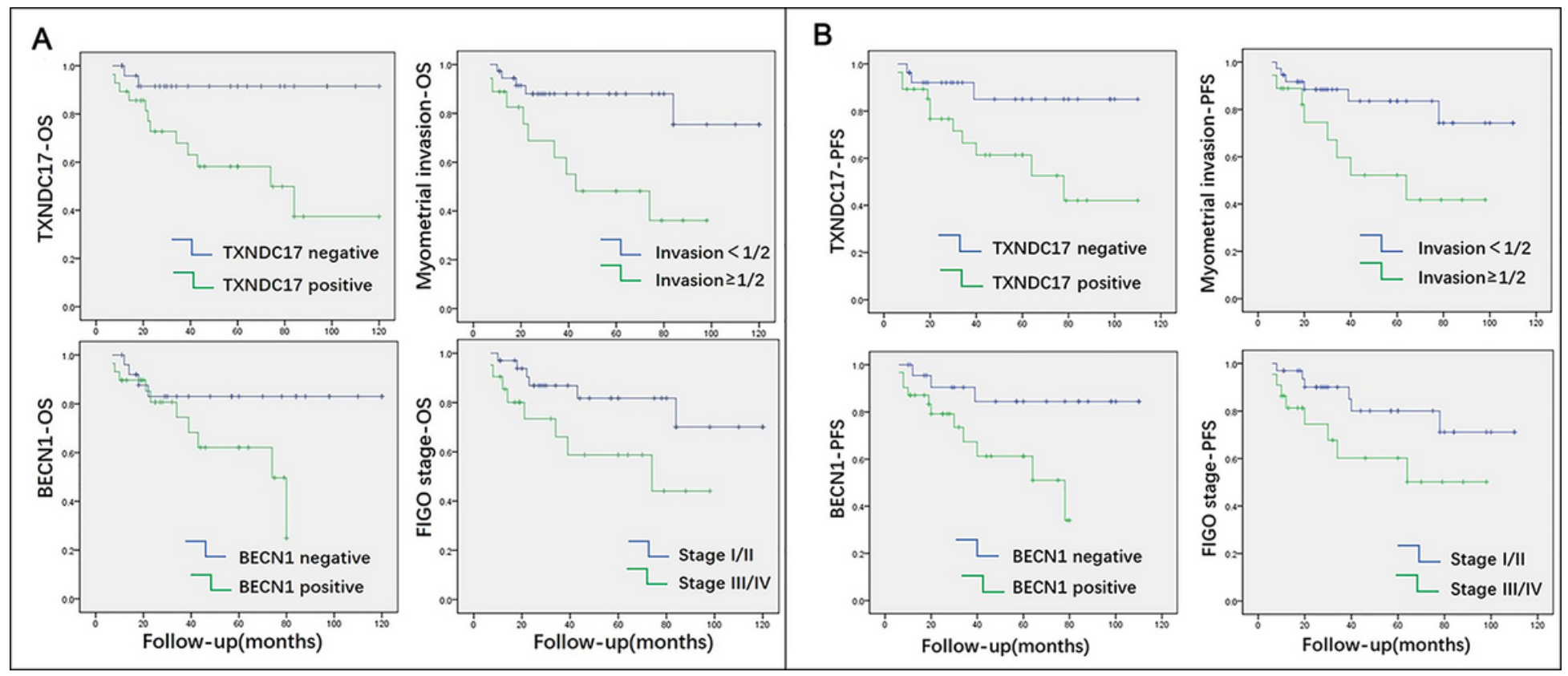

\section{Figure 2}

Kaplan-Meier survival curves for PFS and OS in UPSC patients: (a) Overall survival (OS) based on TXNDC17 expression ( $P=0.008), B E C N 1$ expression $(P=0.047)$, myometrial invasion $(P=0.006)$ and FIGO stage ( $P=0.047)$; (b) Progression-free survival (PFS) based on TXNDC17 expression ( $P=0.028), B E C N 1$ expression $(P=0.030)$, myometrial invasion $(P=0.019)$ and FIGO stage $(P=0.042)$. OS, Overall survival; PFS, Progression-free survival; UPSC, Uterine Papillary Serous Carcinoma; TXNDC17, thioredoxin domain containing 17 protein; BECN1, Beclin 1 protein; FIGO, International Federation of Gynecology and Obstetrics.
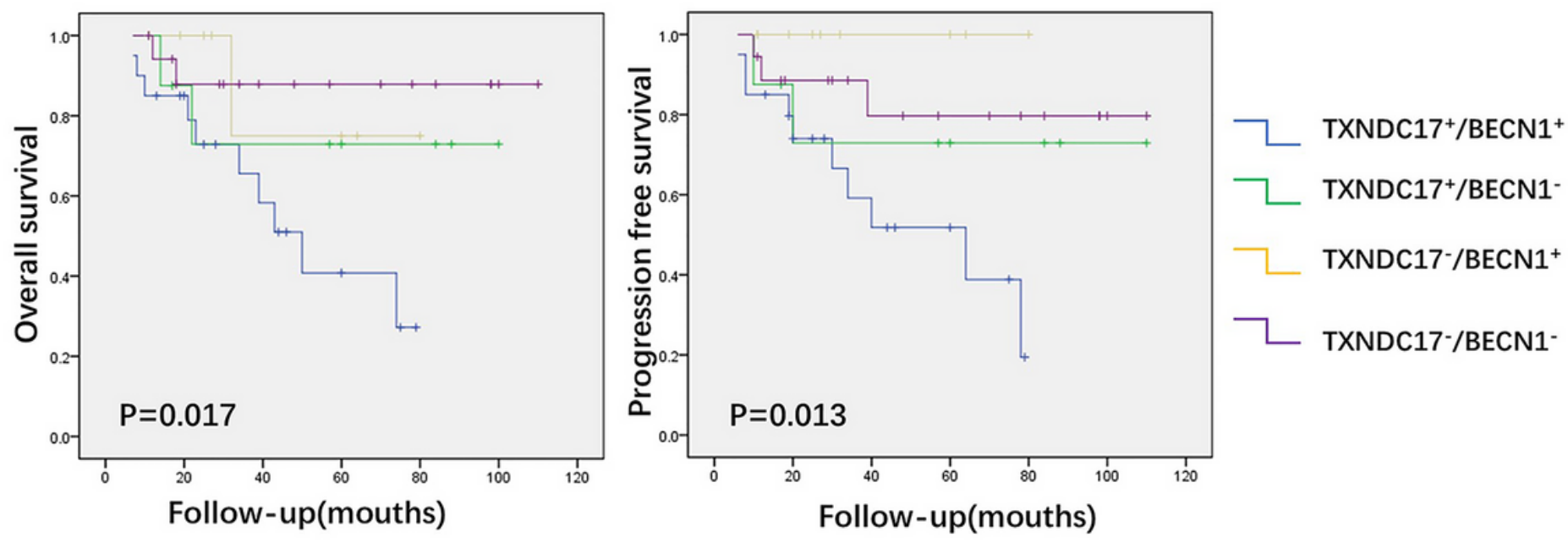

Figure 3

Combined analysis of TXNDC17 and BECN1 expression in the prognostic value of patients with UPSC using Kaplan-Meier survival curves. TXNDC17, thioredoxin domain containing 17 protein; BECN1, Beclin 1 protein; UPSC, uterine papillary serous carcinoma. 


\section{Supplementary Files}

This is a list of supplementary files associated with this preprint. Click to download.

- Table1.png

- Table2.jpeg

- Table3.jpeg

- Table4.jpeg

- Table5.png 